\section{Reply to Dr. Beauchesne}

\author{
Melody Lun • Edwin Lok - Shiva Gautam • \\ Erxi Wu $\cdot$ Eric T. Wong
}

Accepted: 1 August 2012/Published online: 21 August 2012

(C) Springer Science+Business Media, LLC. 2012

\section{To the editor}

We thank Dr. Beauchesne's comment [1] regarding our work on extracranial glioblastoma [2] and, hereby, address the issues raised by him. First, the following search terms were used in the selection of published literature: (i) extracranial glioblastoma, and (ii) extracranial metastasis glioblastoma. Using these terms we identified 88 cases of this disorder. However, as Dr. Beauchesne pointed out, there are other nomenclatures that describe this disorder, most notably extra-neural glioblastoma. In our opinion, extracranial glioblastoma is a more commonly used, as well as a more appropriate, term to describe this type of metastasis outside of the cranium. Quite often, only the end organ involved is noted in the literature. When the term extra-neural is used, it excludes involvement of the peripheral nervous system. However, we think that glioblastoma from the brain or the spinal cord spreading to the peripheral nervous system would still offer a very important insight into the biology of extracranial glioblastoma. Therefore, the nomenclature of

M. Lun · E. Lok · E. T. Wong (

Department of Neurology, Brain Tumor Center \& NeuroOncology Unit, Beth Israel Deaconess Medical Center, Harvard

Medical School, Boston, MA 02215, USA

e-mail: ewong@bidmc.harvard.edu

\section{Lun}

Modular Medical Integrated Curriculum Boston University,

Boston, MA, USA

S. Gautam

Division of Biostatistics, Beth Israel Deaconess Medical Center, Harvard Medical School, Boston, MA, USA

\section{E. Wu}

Department of Pharmacological Sciences, North Dakota State University, Fargo, ND, USA extracranial glioblastoma requires further definition and a consensus needs to be adopted for it use in describing this phenomenon. Second, although our data only included 88 published literatures, and according to Dr. Beauchesne this is underrepresentative to the overall literature of extracranial glioblastoma, our findings are still relevant. Significantly, we found that there was a lead-time bias in the survival of patients as a result of the introduction of modern neuroimaging, like CT and MRI. Furthermore, patients with lung metastases have a poorer prognosis than those with liver or neck metastases. Clearly, our findings are provocative and remain to be validated in a larger sample size. Lastly, it is an affirmation that our work is important when our discordant findings strike such a nerve in our colleague across the Atlantic. Traditionally, extracranial glioblastoma is viewed as an anomaly by neuro-oncologists. However, when we view it within the context of immunological surveillance and immune editing of metastases, it becomes clear that extracranial metastasis of glioblastoma is no different from systemic malignancies that metastasize to the brain. It also dispels the dogma that glioblastoma never goes outside of the brain - we just have not looked hard enough. Therefore, I agree with Dr. Beauchesne that more research needs to be done to determine the fundamental biology of extracranial glioblastoma.

\section{References}

1. Beauchesne P (2012) Letter to the editor: the natural history of extra-cranial metastasis from glioblastoma multiform. J Neurooncol. doi:10.1007/s11060-012-0921-5

2. Lun M, Lok E, Gautam S, Wu E, Wong ET (2011) The natural history of extracranial metastasis from glioblastoma multiforme. J Neurooncol 105:261-273. doi:10.1007/s11060-011-0575-8 